\title{
BRIEF PAPER Surface Clutter Suppression with FDTD Recovery Signal for Microwave UWB Mammography*
}

\author{
Kazuki NORITAKE $^{\dagger}$, Nonmember and Shouhei KIDERA ${ }^{\dagger a)}$, Member
}

\begin{abstract}
SUMMARY Microwave mammography is a promising alternative to $\mathrm{X}$-ray based imaging modalities, because of its small size, low cost, and cell-friendly exposure. More importantly, this modality enables the suppression of surface reflection clutter, which can be enhanced by introducing accurate surface shape estimations. However, near-field measurements can reduce the shape estimation accuracy, due to a mismatch between the reference and observed waveforms. To mitigate this problem, this study incorporates envelope-based shape estimation and finite-difference timedomain (FDTD)-based waveform correction with a fractional derivative adjustment. Numerical simulations based on realistic breast phantoms derived from magnetic resonance imaging (MRI) show that the proposed method significantly enhances the accuracy of breast surface imaging and the performance of surface clutter rejection.

key words: microwave ultra wide-band (UWB) mammography, envelope method, FDTD-based waveform correction
\end{abstract}

\section{Introduction}

According to the World Cancer Research Fund (WCRF), breast cancer is the most widely diagnosed cancer in women worldwide [1]. Although X-ray mammography is the most commonly used screening technique for the detection of malignant tumors, this method damages the cells and involves measurements of the human breast under high compression; resulting in insufficient participation rates, particular in young women. By contrast, ultrasound-based imaging modalities enable safe tumor investigation; however, these techniques require contact measurements, and the detection accuracy is strongly dependent on the skill of the operator. As an alternative, microwave ultra-wideband (UWB) mammography provides several advantages over existing techniques: it is portable and non-contact, involves cell-friendly measurements, and can be implemented at a low cost.

A number of investigations and studies have shown significant contrast between the dielectric properties of normal tissues (typically adipose) and malignant tumors. This electric contrast has triggered the development of microwave mammography, and various imaging algorithms have been developed for cancer detection, such as beamforming (delay and sum (DAS)) [2] and inverse scattering approaches [3].

\footnotetext{
Manuscript received March 20, 2019.

Manuscript revised May 2, 2019.

Manuscript publicized July 17, 2019.

${ }^{\dagger}$ The authors are with Graduate School of Informatics and Engineering, The University of Electro-Communications, Chofu-shi, 182-8585 Japan.

*This research and development work was supported by the MIC/SCOPE \#162103102.

a) E-mail: kidera@ee.uec.ac.jp

DOI: 10.1587/transele.2019ECS6006
}

However, the methods employed to generate high-contrast images of malignant tumors require pre-processing to eliminate skin reflection components, which have a considerably larger signal strength than the tumor response. Some studies have focused on surface reflection rejection, utilizing methods based on time-gating [2], singular value decomposition (SVD) [4], entropy based time windowing [5], and others [6]. However, in the above-mentioned methods, the target tumor response may be suppressed, particularly for cases in which the tumor is located close to the skin surface. To address this issue, some studies have incorporated the breast surface imaging to enhance the clutter rejection performance [7], and in the laser-based techniques e.g. [8], it is difficult to adjust the microwave and laser sensors. For example, one method [9] incorporates the Envelope-based shape estimation [10] and the FDTD-based signal recovery for surface clutter rejection.

However, it has been demonstrated that the reconstruction accuracy of the skin reflection signal is strongly dependent on a prior shape estimation for the outer surface of the breast. This dependence arises because the skin reflection waveform is often mismatched with the reference waveform for a range extraction filter (e.g. matched filter), which includes only a far-field radiation component because of the near-field measurement, where the distance between the antenna and the skin surface is smaller than the measurement wavelength. This mismatch causes a non-negligible error in the matched-filter-based range estimation and leads to inaccurate shape reconstruction.

To resolve this problem, this study proposes a direct compensation scheme for breast boundary estimation errors resulting from the Envelope method by focusing on the time-shift between the observed and FDTD-recovered signals. In addition, this method introduces a fractional derivative adjustment for the FDTD-recovered signal during the clutter suppression stage. Numerical simulation results obtained using realistic MRI-derived numerical phantoms demonstrate that the proposed method considerably enhances the accuracy of breast surface imaging, which significantly contributes to the surface reflection rejection ratio.

\section{Observation Model}

Figure 1 shows the observation model used in this study. A system comprising a transmitting antenna and a receiving antenna rotates around to scan its surface and records the scattered electric field at each observation point (assuming 


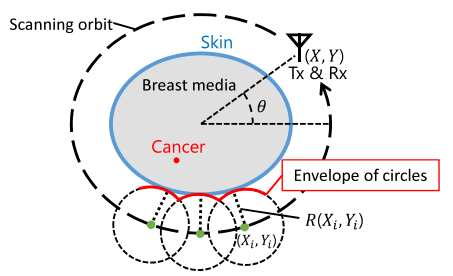

Fig. 1 Observation model and Envelope based boundary reconstruction scheme.

a monostatic observation). The breast medium mainly comprises skin, adipose, and fibro-glandular tissues, which have lossy, dispersive, and isotropic dielectric properties. Here, $s(X, Y, t)$ is defined as the electric field recorded when the transmitting and receiving antennae are located at $(x, y)=$ $(X, Y)$ at time $t$.

\section{Conventional Methods for Clutter Suppression}

A number of methods have been developed to suppress skin reflection [2], in which the skin reflection signal is reconstructed by averaging measurement of signals recorded at different sensor locations. However, such methods risk suppressing not only the skin's reflection but also the tumor response. To avoid over-suppression, one method [9] introduced FDTD-based recovery for skin reflection, which involves prior estimation of the surface shape of the skin using the Envelope method [10]. In the Envelope method, the skin surface boundary is expressed as an outer envelope of circles, the envelope is defined as curve that is tangent to each circle, namely, a group of equi-phase curves. The center of the antenna's location $(X, Y)$ and the radius of the measured range $\hat{R}(X, Y)$, is extracted from the local maximum of the output of the filter (e.g. matched filter) using a specific reference signal. Figure 1 shows the principle of envelope-based surface extraction. Using the breast boundary obtained by the Envelope method [9], the skin reflection signal, which is defined as $\hat{s}(X, Y, t)$, is recovered using the FDTD method by assuming a homogeneous medium with averaged dielectric properties for the breast and skin tissues. The clutter eliminated signal $\tilde{s}_{\text {conv }}(X, Y, t)$ is then formed as;

$$
\tilde{s}_{\text {conv }}(X, Y, t)=s(X, Y, t)-\frac{\max _{t} s(X, Y, t)}{\max _{t} \hat{s}(X, Y, t)} \hat{s}(X, Y, t) .
$$

Although this method can potentially resolve the problem of over-suppression associated with the approach in [2], the actual suppression performance is strongly dependent on the accuracy of the extracted breast boundary. In microwave mammography, the antennae and breast surface are generally closely located within the central wavelength of the transmitted pulse. Thus, in this near-field observation, the reflection signal waveform is considerably different from the assumed reference waveform, which assumes only a far field component. This waveform deformation leads to an inaccurate range estimation, and reducing the accuracy for Envelope-based breast boundary extraction.

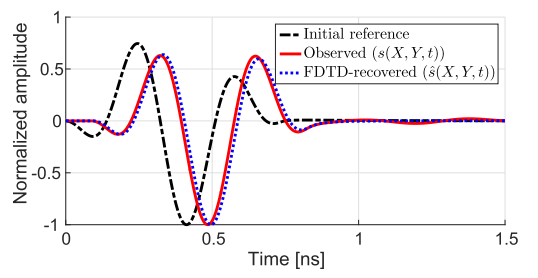

Fig. 2 Waveform comparison among observed signal $s(X, Y, t)$, initial reference signal and FDTD-recovered signal $\hat{s}(X, Y, t)$.

\section{Proposed Method}

To enhance the accuracy of breast surface estimation, this study produces a direct range compensation method using the FDTD-recovered waveform. The proposed method is based on the premise that the FDTD-recovered signal includes the near-field component because of the proximity between the skin and the antennae, which depends on the source and observation locations $(X, Y)$. Therefore, the similarity between the FDTD-recovered and observed waveforms should be improved. Figure 2 shows a comparison of waveforms for the observed, initial reference, and FDTDrecovered signals $\hat{s}(X, Y, t)$ for specific breast media and observation points, where $\hat{s}(X, Y, t)$ is computed by using the group of $\hat{R}(X, Y)$ through the Envelope based surface shape estimation. Note that, the initial reference signal is usually obtained for far-field situations, where the transmitter and the receiver are separated by multiple wavelengths. This figure demonstrates that the initial reference waveform differs slightly from the observed signal even when a timeshift compensation is employed. By contrast, the FDTDrecovered signal matches well with the actual observation signal. By exploiting the above relationship, this method updates the range between skin's surface and the antenna location $(X, Y)$ denoted as $\tilde{R}(X, Y)$;

$$
\tilde{R}(X, Y)=\hat{R}(X, Y)+\mathrm{c} \Delta \tau(X, Y) / 2 .
$$

$\mathrm{c}$ is the speed of light in air, and $\Delta \tau(X, Y)$ is calculated as;

$$
\Delta \tau(X, Y)=\underset{\tau}{\arg \max }[s(X, Y, t) \star \hat{s}(X, Y, t)](\tau),
$$

where $\star$ denotes the cross-correlation operator. The boundary of the breast surface is also updated by the Envelope method using the group of $\tilde{R}(X, Y)$. In the next stage of clutter suppression using the FDTD-recovered signal, this method introduces adjustment of the fractional-derivativebased waveform, using a parameter $\alpha$, the adjusted signal is expressed as;

$$
\hat{s}_{\text {up }}(X, Y, t, \alpha)=\mathcal{F}^{-1}\left[(j \omega)^{\alpha} \hat{S}_{\text {up }}(X, Y, \omega)\right],
$$

where $\hat{S}_{\text {up }}(X, Y, \omega)=\mathcal{F}\left[\hat{s}_{\text {up }}(X, Y, t)\right]$ and $\mathcal{F}$ denotes the Fourier transform operator. Here, $\hat{s}_{\text {up }}(X, Y, t)$ is generated by the FDTD method, where the breast area is updated by the Envelope method using $\tilde{R}(X, Y)$ in Eq. (2). In the proposed method, the FDTD calculations are performed twice; first, 
$\hat{s}(X, Y, t)$ is obtained, and then, $\hat{s}_{\text {up }}(X, Y, t)$ is calculated. The clutter eliminated signal is then updated as;

$$
\tilde{s}_{\text {prop }}(X, Y, t)=s(X, Y, t)-\hat{A} \hat{s}_{\text {up }}(X, Y, t-\hat{\tau}, \hat{\alpha}),
$$

where $\hat{A}, \hat{\tau}$ and $\hat{\alpha}$ are determined as;

$$
\begin{aligned}
& (\hat{A}, \hat{\tau}, \hat{\alpha}) \\
& =\underset{(A, \tau, \alpha)}{\arg \min } \int_{T_{r}}^{T_{r}+T_{W}}\left|s(X, Y, t)-A \hat{s}_{\mathrm{up}}(X, Y, t-\tau, \alpha)\right|^{2} d t,
\end{aligned}
$$

where, $T_{r}=2 \tilde{R}(X, Y) / \mathrm{c}$ corresponds to the rise time of $s(X, Y, t)$, and $T_{W}$ denotes the temporal window length, which is determined by considering the effective pulsewidth of the transmitted signal. Equation (6) optimizes the surface reflection waveform for a finite time-gate from the first arrival time.

\section{Evaluation with Numerical Breast Phantoms}

Our method was investigated using simulated array measurements of realistic breast phantoms derived from MRI scans of healthy women [11]: Class 2 (Breast ID 010204, Scattered Fibroglandular) phantom was also employed, and cancer cells were intentionally added to this phantom. The transmitting signal formed a raised-cosine modulated pulse with a central frequency of $2.45 \mathrm{GHz}$, and a bandwidth of $2.7 \mathrm{GHz}$. A set of transmitting and receiving antennae, located in the air, scans along the circumference of the breast medium for 31 uniform samples. An excited current is applied at a point source transmitter, namely a short dipole antenna; this antenna model has been experimentally demonstrated as reported in [12]. Frequency-dependent complex permittivities for the breast phantoms were modeled using single-pole Debye models. The scattered electric field was calculated by the FDTD method with the single-pole Debye model (Matlab code was provided by the cross-disciplinary electromagnetics laboratory led by Prof. S.C. Hagness at the University of Wisconsin, Madison). We added Gaussian white noise to the received signals, where the noise power level is $70 \mathrm{~dB}$ lower than that of the source. This value is a conservative estimate for the expected noise floor of a data acquisition system. With this noise floor, the signal-to-noise ratio (SNR), where the power of the backscatter signal is regarded as $\mathrm{S}$, is approximately $16 \mathrm{~dB}$. To clarify the suppression performance of each method, the following two cases were investigated. CASE 1 assumed a dispersive but homogeneous breast medium except for skin and cancer tissues, which enabled us to focus solely on the separation of the skin and cancer reflections. CASE 2 assumed a realistic heterogeneous case directly derived from the database [11]. Figure 3 presents plots of the Debye parameter, $\Delta \epsilon$, which is defined as the difference between the relative permittivity from zero to infinite frequencies, for CASES 1 and 2. For CASE 1, the parameters for breast medium (adipose dominant tissue) in the Debye model are set as $\sigma=0.07[\mathrm{~S} / \mathrm{m}]$, $\epsilon(f=0)=7.2$, and $\epsilon(f=\infty)=4.0$, and those for the

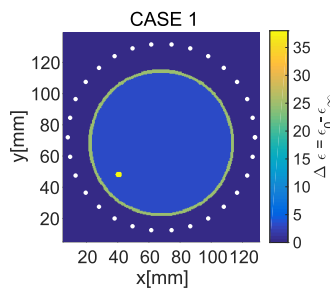

(a)

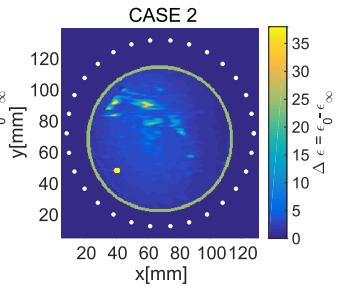

(b)
Fig. 3 The maps of the Debye parameter $\Delta \epsilon(($ a) CASE 1 and (b) CASE 2 (MRI-derived Class 2)).

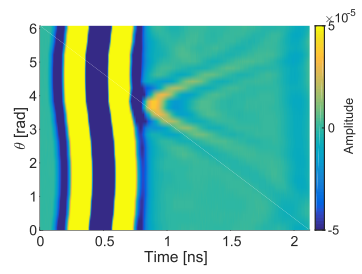

(a)

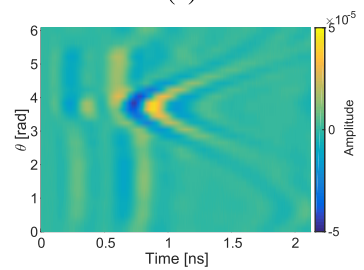

(c)

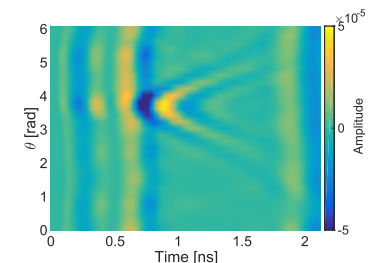

(b)

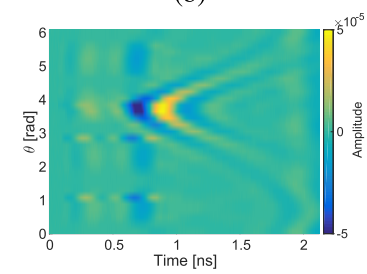

(d)
Fig. 4 Signal subtraction results in CASE 1. (a) Original signal. Clutter eliminated signals by the method I (b), the method II (c) and the proposed method (d).

cancer tissue are set as $\sigma=0.8[\mathrm{~S} / \mathrm{m}], \epsilon(f=0)=58.0$, and $\epsilon(f=\infty)=20.0$, as reported in [11]. Here, we denote Method I as the conventional method described in [9], and Method II as that derived from [2], where the skin reflection signal is modeled by averaging the signals recorded at different sensor locations after compensating each time-shift using each peak-shift of cross-correlation functions among those signals. Note that, to clarify the advantage of the proposed method over the Method II, that uses a simple mean trace subtraction, the center of breast is slightly shifted from the center of circular arrays in both cases. In this case, then, the near-field effect, incurring waveform deformation, is slightly different at each antenna location, which is not compensated by the Method II. In the proposed method, the Nelder-Mead simplex method was used for the optimization problem in Eq. (6), and with $T_{W}=0.75 \mathrm{~ns}$.

Figure 4 shows the signal distribution for the observed and clutter eliminated signals for CASE 1 for each method. Figure 4 shows that the proposed method efficiently suppressed the skin reflection, particularly for the case in which the skin and cancer tissues were in close proximity. The proposed method was superior because the method in [9] does not update the shape of the breast or the clutter signal using Eqs. (2) or (4). We confirmed that the suppression achieved by a scaling term was not sufficient, but was required to compensate the frequency dependency denoted 


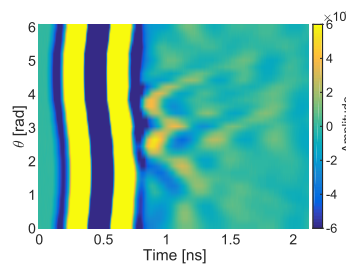

(a)

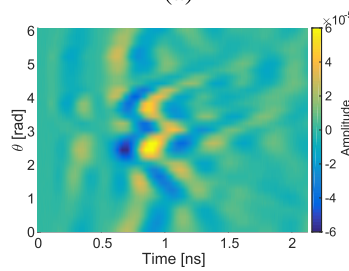

(c)

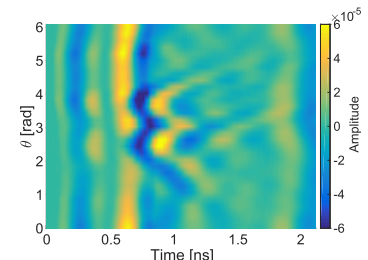

(b)

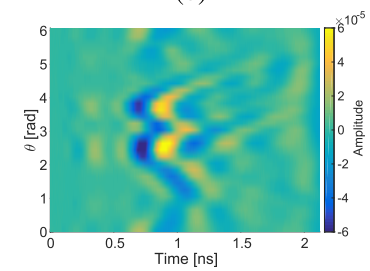

(d)
Fig. 5 Signal subtraction results in CASE 2. (a) Original signal. Clutter eliminated signals by the method I (b), the method II (c) and the proposed method (d).

Table 1 RMSE and PPRR at SNR $=16 \mathrm{~dB}$.

\begin{tabular}{|c|c||c|c|c|}
\hline & & Method I & Method II & Proposed \\
\hline \hline \multirow{2}{*}{ RMSE[mm] } & CASE 1 & \multicolumn{2}{|c|}{2.1} & 0.5 \\
\cline { 2 - 5 } & CASE 2 & \multicolumn{2}{|c|}{2.6} & 0.6 \\
\hline \multirow{2}{*}{ PPRR[dB] } & CASE 1 & -28.5 & -38.1 & -42.8 \\
\cline { 2 - 5 } & CASE 2 & -21.8 & -32.6 & -37.8 \\
\hline
\end{tabular}

in Eq. (4), namely, the degree of the fractional derivative as $\alpha$ should be optimized. Figure 5 shows the same results presented in Fig. 4, for CASE 2. The results demonstrate that the proposed method achieves superior performance in terms of surface shape estimation and skin reflection suppression without sacrificing the tumor response. A quantitative analysis was then conducted as follows. The error in the surface boundary estimation was defined as the minimum distance between the boundary points extracted using the Envelope method and the actual boundary. The peak-topeak response ratio (PPRR), introduced in [6], was applied to assess the clutter suppression performance. The PPRR is defined as the ratio of peak-to-peak values before and after the suppression method is applied, within the appropriate time span, which is defined as $0.186 \mathrm{~ns}$. Table 1 presents the root mean square errors (RMSE) for the surface shape estimation and the PPRRs, verifying that the proposed method substantially enhances the surface shape estimation accuracy, thus increasing the PPRR and improving the identification of cancer tissues.

\section{Conclusion}

This study proposed a clutter suppression method for addressing skin reflection by compensating for the breast surface shape using FDTD-based waveform reconstruction. In addition, a fractional derivative model of the FDTDreconstructed waveform is adopted for accurate clutter rejection. Investigations using realistic numerical phantoms of breast medium showed that the proposed method sub- stantially enhances the breast surface reconstruction accuracy and provides significant improvements in the PPRR for both homogeneous and heterogeneous media. Although, the proposed method requires a priori knowledge of average complex permittivity of breast media for FDTD-based waveform reconstruction, accurate values can be retrieved from a number of studies, e.g., [2]. Naturally, the performance of the proposed method depends on the assumed dielectric properties, which are not accurately known in practical situations. To address this problem, we are planning to combine our proposed method with a permittivity reconstruction method, e.g., an inverse scattering algorithm. We note that the study in [2] assumes that the antenna is placed directly on the breast skin, which differs from the observation model in this study. However, even for the model assumed in [2], surface clutter suppression is required, and the simulations in this study demonstrated that the proposed method attains more effective clutter suppression, compared with the approach in [2], by excluding the cancer response.

\section{References}

[1] F. Bray, J. Ferlay, I. Soerjomataram, R.L. Siegel, L.A. Torre, and A. Jemal, "Global cancer statistics 2018: GLOBOCAN estimates of incidence and mortality worldwide for 36 cancers in 185 countries," Cancer Jnl. for Clinicians, vol.68, no.6, pp.394-424, Dec. 2018.

[2] E.J. Bond, X. Li, S.C. Hagness, and B.D. Van Veen, "Microwave imaging via space-time beamforming for early detection of breast cancer," IEEE Trans. Antennas Propagat., vol.51, no.8, pp.1690-1705, Aug. 2003.

[3] F. Gao, B.D. Van Veen, and S.C. Hagness, "Sensitivity of the distorted born iterative method to the initial guess in microwave breast imaging," IEEE Trans. Antennas Propagat., vol.63, no.8, pp.3540-3547, Aug. 2015.

[4] F. Abujarad, A. Jostingmeier, and A. Omar, "Clutter removal for landmine using different signal processing techniques," Proc. Int. Conf. Ground Penetrating Radar, pp.697-700, 2004.

[5] W. Zhi and F. Chin, "Entropy-based time window for artifact removal in UWB imaging of breast cancer detection," IEEE Signal Process. Lett., vol.13, no.10, pp.585-588, Oct. 2006.

[6] M.A. Elahi, M. Glavin, E. Jones, and M. O'Halloran, "Artifact removal algorithms for microwave imaging of the breast," Progress In Electromagnetics Research, vol.141, pp.185-200, 2013.

[7] D.W. Winters, J.D. Shea, E.L. Madsen, G.R. Frank, B.D. Van Veen, and S.C. Hagness, "Estimating the breast surface using UWB microwave monostatic backscatter measurements," IEEE Trans. Biomed. Eng., vol.55, no.1, pp.247-256, Jan. 2008.

[8] T.C. Williams, J. Bourqui, T.R. Cameron, M. Okoniewski, and E.C. Fear, "Laser surface estimation for microwave breast imaging systems," IEEE Trans. Biomed. Eng., vol.58, no.5, pp.1193-1199, May 2011.

[9] F. Endo and S. Kidera, "Accuracy enhanced beamorming method based on envelope surface extraction for non-contact UWB breast cancer radar," IEEE Proc. ISAP, Oct. 2016.

[10] S. Kidera, T. Sakamoto, and T. Sato, "A robust and fast imaging algorithm with an envelope of circles for UWB pulse radars," IEICE Trans. Commun., vol.E90-B, no.7, pp.1801-1809, July 2007.

[11] UWCEM, "Numerical breast phantom repository," URL:https://uwcem.ece.wisc.edu/phantomRepository.html

[12] L.M. Neira, B.D. Van Veen, and S.C. Hagness, "High-resolution microwave breast imaging using a 3-D inverse scattering algorithm with a variable-strength spatial prior constraint," IEEE Trans. Antennas Propagat., vol.65, no.11, pp.6002-6014, Sept. 2017. 